Research Article

\title{
Two Classes of Infrasoft Separation Axioms
}

\author{
Tareq M. Al-shami $\mathbb{D D}^{1}$ and Jia-Bao Liu $\mathbb{D}^{2}$ \\ ${ }^{1}$ Department of Mathematics, Sana'a University, Sana'a, Yemen \\ ${ }^{2}$ School of Mathematics and Physics, Anhui Jianzhu University, Hefei 230601, China \\ Correspondence should be addressed to Tareq M. Al-shami; tareqalshami83@gmail.com
}

Received 21 July 2021; Accepted 26 October 2021; Published 20 November 2021

Academic Editor: Feng Feng

Copyright (c) 2021 Tareq M. Al-shami and Jia-Bao Liu. This is an open access article distributed under the Creative Commons Attribution License, which permits unrestricted use, distribution, and reproduction in any medium, provided the original work is properly cited.

\begin{abstract}
One of the considerable topics in the soft setting is the study of soft topology which has enticed the attention of many researchers. To contribute to this scope, we devote this work to investigate two classes of separation axioms with respect to the distinct ordinary elements through one of the generalizations of soft topology called infrasoft topology. We first formulate the concepts of infra$t p$-soft $T_{j}$ using total belong and partial nonbelong relations and then introduce the concepts of infra-t $t$-soft $T_{j}$-spaces using total belong and partial nonbelong relations. To illustrate the relationships between them, we provide some examples. We discuss their fundamental properties and study their behaviors under some special types of infrasoft topologies. An extensive discussion is given for the transmission of these two classes between infrasoft topology and its parametric infratopologies. In the end, we demonstrate which ones have topological and hereditary properties, and we show their behaviors under the finite product of soft spaces.
\end{abstract}

\section{Introduction}

One of the powerful mathematical approaches to cope with uncertain problems is the soft set. It was proposed in 1999 by Molodtsov [1] who explained its applications to different areas such as smoothness of functions, theory of measurement, game theory, Riemann integration, operations research, etc. In 2002, soft sets were successfully applied to handle decision-making problems by Maji et al. [2]. They introduced some operations and operators on soft sets such as their soft intersection and union and the complement of a soft set in [3]. Despite the weakness of some concepts and results in these early references, they form the essential start point of soft set theory. To keep some properties and results of crisp set theory, Ali et al. [4] displayed new types of these operations and operators. Novel types of them were established such as lower and upper soft equality [5], $g f$-soft union [6], and $T$-soft subset and $T$-soft equality [7]. Since the advent of soft sets, many authors applied successfully to address problems in some disciplines such as computer science [8], decision-making [9, 10], and medical science $[11,12]$. These applications prove the adequacy of soft sets to treat and model a lot of real-life issues.
In 2011, Shabir and Naz [13] defined soft topologies as hybridization of classical topology and soft sets. Then, numerous researchers have discussed the topological concepts, properties, and results in soft topologies; see [14-17]. Soft point $[18,19]$ is an essential concept in soft set theory; it represents the soft version of an ordinary element. Some types of soft topologies called enriched and extended soft topologies are studied [19].

Some topological properties are kept under conditions weaker than topology's conditions; also, some phenomena are described under structures relaxing a topology. The structures of suprasoft topology [20] and infrasoft topology [21] have been born with these goals. They have become two of the most interesting developments of soft topology in recent years. Supratopology is a class of subsets that extend the concept of topological space by dispensing with the postulate that the class is closed under finite intersections, whereas infratopology is a class of subsets that extend the concept of topological space by dispensing with the postulate that the class is closed under arbitrary unions. Generalized soft topology [22] and soft weak structure are other generalizations of soft topology. Recently, the concepts of 
compactness and connectedness have been studied through the frame of infrasoft topology in [23, 24], respectively.

Another technique of generalizing soft topology was introduced by combining other structures with a soft topology. In this regard, Ittanagi [25] initiated the concept of soft bitopology.

Our contribution to this field concerns the analysis of what type of "separation axioms" are meaningful in the study of infrasoft topology. As was the case of classical topology, soft separation axioms are among the most interesting and substantial ideas in soft topology. They form a tool to establish more restricted (and wider) classes of well-behaved soft spaces. It should be noted that a large variety of separation axioms in the soft structures is attributed to two factors. One is the distinguished objects that we intend to separate: they can be either ordinary points or soft points. The other is the type of belongingness and nonbelongingness relations that we require in the definitions: they can be either partial or total. For more details about this subject, see [26], which investigated new sorts of separation axioms on suprasoft topologies, and [10,27], which studied two kinds of soft $T_{i}$-spaces on soft topologies. In [28], the researchers presented soft separation axioms with respect to distinct soft points. Terepeta [29] discussed soft separation axioms using their counterparts in classical topological spaces. Singh and Noorie [30] conducted a comparative study among some soft separation axioms published in the literature.

In this article, we note that many properties of soft topological spaces are still valid on infrasoft topological spaces and initiating examples that show some relationships between certain topological concepts is easier on infrasoft topological spaces. Therefore, we aim in this paper to perform an exhaustive analysis of infrasoft topological spaces.

The organization of this manuscript is as follows. Section 2 mentions some definitions and properties related to soft set theory and infrasoft topology. Section 3 initiates the concepts of infra-t $p$-soft $T_{i}$-spaces $(i=0,1,2,3,4)$ and studies basic properties. Section 4 defines the concepts of infra$t$-soft $T_{i}$-spaces $(i=0,1,2,3,4)$ and discusses main properties. Elucidative examples are supplied to validate the obtained relationships and results. Ultimately, Section 5 outlines the paper's achievements and proposes some future works.

\section{Preliminaries}

The concepts and results that we need in this paper are recalled in this section.

2.1. Soft Set Theory. The concepts and results of soft set theory that we need in this paper are mentioned in this part.

Definition 1 (see [1]). A map $E$ from $\Sigma$ which is set of parameters to the power set $2^{\mathbb{Y}}$ of $\mathbb{Y}$ is called a soft set over $\mathbb{Y}$. It is denoted by $E_{\Sigma}$ and identified as $E_{\Sigma}=\left\{(\sigma, E(\sigma)): \sigma \in \Sigma\right.$ and $\left.E(\sigma) \in 2^{\mathbb{Y}}\right\}$.

The set of all soft sets over $\mathbb{Y}$ with $\Sigma$ as a set of parameters is symbolized by $S\left(\mathbb{Y}_{\Sigma}\right)$.
Definition 2 (see [4]). The complement of a soft set $E_{\Sigma}$, symbolized by $E_{\Sigma}^{c}$, is given by $E_{\Sigma}^{c}=\left(E^{c}\right)_{\Sigma}$, where $E^{c}: \Sigma \longrightarrow 2^{\mathbb{V}}$ is a map defined by $E^{c}(\sigma)=\mathrm{Y}, E(\sigma)$ for each $\sigma \in \Sigma$.

Definition 3 (see [2]). If the image of each parameter of $\Sigma$ under a map $E: \Sigma \longrightarrow \mathbb{Y}$ is the empty set, then $E_{\Sigma}$ is called the null soft set over $\mathbb{Y}$. Its complement is called the absolute soft set. The notations $\Phi$ and $\widetilde{\mathbb{Y}}$, respectively, denote the null and absolute soft sets.

Definition 4 (see $[18,19])$. If the image of one parameter, say $\sigma$, under a map $P: \Sigma \longrightarrow \bigvee$ is a singleton set, say $\{\nu\}$, and the image of each parameter $\sigma^{\prime} \in \Sigma,\{\sigma\}$ is the empty set, then a soft set $P_{\Sigma}$ is called a soft point over $\mathbb{Y}$. It is briefly symbolized by $P_{\sigma}^{v}$.

Definition 5 (see [4]). The intersection of two soft sets $E_{\Omega}$ and $F_{\Gamma}$ over $\mathbb{Y}$, symbolized by $E_{\Omega} \widetilde{\cap} F_{\Gamma}$, is a soft set $H_{\Sigma}$, where $\Sigma=\Omega \cap \Gamma \neq \varnothing$, and a map $H: \Sigma \longrightarrow 2^{\mathbb{V}}$ is given by $H(\sigma)=$ $E(\sigma) \cap F(\sigma)$ for each $\sigma \in \Sigma$.

Definition 6 (see [4]). The union of two soft sets $E_{\Omega}$ and $F_{\Gamma}$ over $\mathbb{Y}$, symbolized by $E_{\Omega} \widetilde{\cup} F_{\Gamma}$, is a soft set $H_{\Sigma}$, where $\Sigma=$ $\Omega \cup \Gamma$ and a map $H: \Sigma \longrightarrow 2^{\mathbb{Y}}$ is given as follows:

$$
H(\sigma)= \begin{cases}E(\sigma), & \sigma \in \Omega, \Gamma, \\ F(\sigma), & \sigma \in \Gamma, \Omega, \\ E(\sigma) \cup F(\sigma), & \sigma \in \Omega \cap \Gamma .\end{cases}
$$

Definition 7 (see [31]). A soft set $E_{\Sigma}$ is a subset of a soft set $F_{\Omega}$, symbolized by $E_{\Sigma} \widetilde{\subseteq} F_{\Omega}$, if $\Sigma \subseteq \Omega$ and for all $\sigma \in \Sigma$, we have $E(\sigma) \subseteq F(\sigma)$. The soft sets $E_{\Sigma}$ and $E_{\Omega}$ are called soft equal if each is a subset of the other.

Definition 8 (see [13]). A soft set $E_{\Sigma}$ over $\mathbb{V}$ which is defined by $E(\sigma)=\{\nu\}$ for each $\sigma \in \Sigma$ is called a singleton soft set. It is denoted by $\nu_{\Sigma}$.

Definition 9 (see [27]). If all components of a soft set are equal, then we called it a stable soft set.

Definition 10 (see [16]). The Cartesian product of $E_{\Sigma}$ and $H_{\Omega}$, symbolized by $(G \times H)_{\Sigma \times \Omega}$, is defined as $(G \times H)(\sigma, \omega)=E(\sigma) \times H(\omega)$ for each $(\sigma, \omega) \in \Sigma \times \Omega$.

The definition of soft maps given in [32] was reformulated in a way that reduces calculation burden and gives a justification (logical explanation) for some soft concepts such as why we determine that $f_{\eta}$ is injective or surjective according to its two crisp maps $f$ and $\eta$.

Definition 11 (see [33]). Let $f: \mathbb{X} \longrightarrow \mathbb{Y}$ and $\eta: \Sigma \longrightarrow \Omega$ be two crisp maps. A soft map $f_{\eta}$ of $S\left(\mathbb{X}_{\Sigma}\right)$ into $S\left(\mathbb{Y}_{\Omega}\right)$ is a relation such that each soft point in $S\left(\mathbb{X}_{\Sigma}\right)$ is related to one and only one soft point in $S\left(\mathbb{Y}_{\Omega}\right)$ such that $f_{\eta}\left(P_{\sigma}^{v}\right)=P_{\eta(\sigma)}^{f(\nu)}$ for each $P_{\sigma}^{v} \in S\left(\mathbb{X}_{\Sigma}\right)$.

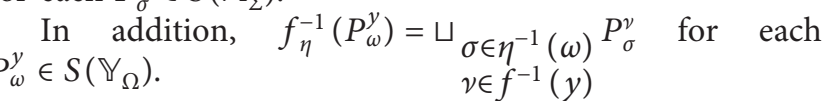


Definition 12 (see [32]). A soft map $f_{\eta}: S\left(\mathbb{Y}_{\Sigma}\right) \longrightarrow S\left(\mathbb{Z}_{\Omega}\right)$ is said to be injective (resp. surjective, bijective) if both $f$ and $\eta$ are injective (resp., surjective, bijective).

Definition 13 (see $[13,27]$ ). For a soft set $E_{\Sigma}$ over $\bigvee$ and $\nu \in \mathbb{Y}$, we say that

(i) $\nu \in E_{\Sigma}$ (it reads as $\nu$ totally belongs to $E_{\Sigma}$ ) if $\nu \in E(\sigma)$ for each $\sigma \in \Sigma$;

(ii) $\nu \notin E_{\Sigma}$ (it reads as $\nu$ does not partially belong to $E_{\Sigma}$ ) if $\nu \notin E(\sigma)$ for some $\sigma \in \Sigma$;

(iii) $\nu \Subset E_{\Sigma}$ (it reads as $\nu$ partially belongs to $E_{\Sigma}$ ) if $\nu \in E(\sigma)$ for some $\sigma \in \Sigma$;

(iv) $\nu \notin E_{\Sigma}$ (it reads as $v$ does not totally belong to $E_{\Sigma}$ ) if $\nu \notin E(\sigma)$ for each $\sigma \in \Sigma$.

Proposition 1 (see [27]). Consider a soft map $f_{\eta}: S\left(\mathbb{Y}_{\Sigma}\right) \longrightarrow S\left(\mathbb{Z}_{\Omega}\right)$ and let $E_{\Sigma}$ and $H_{\Omega}$ be soft sets in $S\left(\mathbb{Y}_{\Sigma}\right)$ and $S\left(\mathbb{Z}_{\Omega}\right)$, respectively. Then the next results hold true:

(i) If $\eta$ is surjective and $\nu \in E_{\Sigma}$, then $f(\nu) \in f_{\eta}\left(E_{\Sigma}\right)$.

(ii) If $\nu \notin E_{\Sigma}$, then $f(\nu) \notin f_{\eta}\left(E_{\Sigma}\right)$ provided that $f$ is injective.

(iii) If $f_{\eta}$ is injective and $\nu \notin E_{\Sigma}$, then $f(\nu) \notin f_{\eta}\left(E_{\Sigma}\right)$.

(iv) If $\mu \in H_{\Omega}$, then $\nu \in f_{\eta}^{-1}\left(H_{\Omega}\right)$ for each $\nu \in f^{-1}(\mu)$.

(v) If $\mu \notin H_{\Omega}$, then $\nu \notin f_{\eta}^{-1}\left(H_{\Omega}\right)$ for each $\nu \in f^{-1}(\mu)$.

(vi) If $\eta$ is surjective and $\mu \notin H_{\Omega}$, then $\nu \notin f_{\eta}^{-1}\left(H_{\Omega}\right)$ for each $\nu \in f^{-1}(\mu)$.

2.2. Infrasoft Topological Spaces. The concepts and results of infrasoft topology that we need in this paper are mentioned in this part.

Definition 14 (see [21]). The collection $\delta$ of soft sets over $\mathbb{Y}$ under a parameters set $\Sigma$ is said to be an infrasoft topology on $\mathbb{Y}$ if it is closed under finite soft intersection and $\Phi \in \delta$.

The triple $(\mathbb{Y}, \delta, \Sigma)$ is called an infrasoft topological space. Every member of $\delta$ is called an infrasoft open set and its relative complement is called an infrasoft closed set. We called $(\mathbb{Y}, \delta, \Sigma)$ stable if all its infrasoft open sets are stable.

Definition 15 (see [21]). We define the infrainterior points and infraclosure points of a soft subset $E_{\Sigma}$ of $(\mathbb{Y}, \delta, \Sigma)$ which are, respectively, denoted by $\operatorname{Int}_{\delta}\left(E_{\Sigma}\right)$ and $\mathrm{Cl}_{\delta}\left(E_{\Sigma}\right)$ as follows:

(i) $\operatorname{Int}_{\delta}\left(E_{\Sigma}\right)$ is the union of all infrasoft open sets contained in $E_{\Sigma}$.

(ii) $\mathrm{Cl}_{\delta}\left(E_{\Sigma}\right)$ is the intersection of all infrasoft closed sets containing $E_{\Sigma}$.

Theorem 1 (see [21]). Let $E_{\Sigma}$ and $F_{\Sigma}$ be two soft subsets of $(\mathbb{Y}, \delta, \Sigma)$. Then,

(i) If $E_{\Sigma} \widetilde{\simeq} F_{\Sigma}$, then $C l_{\delta}\left(E_{\Sigma}\right) \widetilde{\simeq} C l_{\delta}\left(F_{\Sigma}\right)$; (ii) $P_{\sigma}^{v} \in C l_{\delta}\left(E_{\Sigma}\right)$ if and only if $E_{\Sigma} \widetilde{\cap} H_{\Sigma} \neq \Phi$ for each infrasoft open set $F_{\Sigma}$ containing $P_{\sigma}^{v}$.

Proposition 2 (see [21]). Let $(\mathbb{Y}, \delta, \Sigma)$ be an infrasoft topological space. Then the collection $\delta_{\sigma}=\left\{E(\sigma): E_{\Sigma} \in \delta\right\}$ forms an infratopology on $\mathbb{Y}$ for each $\sigma \in \Sigma$.

We called $\delta_{\sigma}$ a parametric infratopology.

Proposition 3 (see [21]). Suppose that $\left\{\delta_{\sigma}\right\}_{\sigma \in \Sigma}$ is a family of crisp infratopologies on $\mathbb{Y}$. Then the family

$\left\{\{(\sigma, E(\sigma)): \sigma \in \Sigma\} \in S\left(\mathbb{Y}_{\Sigma}\right)\right.$ such that $E(\sigma) \in \delta_{\sigma}$ for each $\left.\sigma \in \Sigma\right\}$,

defines an infrasoft topology on $\mathbb{Y}$.

The infrasoft topological space given in the above proposition is called an extended infrasoft topology.

Definition 16 (see [21]). Let $(\mathbb{Y}, \delta, \Sigma)$ be an infrasoft topological space and $\mathbb{Z}$ be a nonempty subset of $\mathbb{Y}$. Then $\delta_{\mathbb{Z}}=$ $\left\{\widetilde{\mathbb{Z}} \cap E_{\Sigma}: E_{\Sigma} \in \delta\right\}$ is called an infrasoft relative topology on $\mathbb{Z}$ and $\left(\mathbb{Z}, \delta_{\mathbb{Z}}, \Sigma\right)$ is called an infrasoft subspace of $(\mathbb{Y}, \delta, \Sigma)$.

Theorem 2 (see $[21])$. Let $\left(\mathbb{Z}, \delta_{\mathbb{Z}}, \Sigma\right)$ be an infrasoft subspace of $(\mathbb{Y}, \delta, \Sigma)$. Then $H_{\Sigma}$ is an infrasoft closed subset of $\left(\mathbb{Z}, \delta_{\mathbb{Z}}, \Sigma\right)$ iff there exists an infrasoft closed subset $F_{\Sigma}$ of $(\mathbb{Y}, \delta, \Sigma)$ such that $H_{\Sigma}=\tilde{\mathbb{Z}} \widetilde{\cap} F_{\Sigma}$

Definition 17 (see [33]). A soft mapping $f_{\varphi}:(\mathbb{Y}, \delta, \Sigma) \longrightarrow(\mathbb{Z}, \theta, \Omega)$ is said to be

(i) Infrasoft continuous if the preimage of any infrasoft open set is an infrasoft open set;

(ii) Infrasoft open (resp., infrasoft closed) if the image of any infrasoft open (resp., infrasoft closed) set is an infrasoft open (resp., infrasoft closed) set;

(iii) An infrasoft homeomorphism if it is bijective, infrasoft continuous, and infrasoft open.

3. Infra- $t p$-Soft $T_{i}$-Spaces $(i==0,1,2,3,4)$

In this section, we display new family of separation axioms, namely, infra-t $p$-soft $T_{i}$-spaces $(i=0,1,2,3,4)$, where $t$ and $p$ are the first letters of total belong and partial nonbelong relations that are used to define these spaces. We explore main properties and supply various examples to elucidate the relationships between them.

Definition 18. $(\mathbb{Y}, \delta, \Sigma)$ is said to be

(i) An infra-tp-soft $T_{0}$-space if for every $\nu \neq \mu \in \mathbb{Y}$, there exists an infrasoft open set $E_{\Sigma}$ such that $\nu \in E_{\Sigma}$ and $\mu \notin E_{\Sigma}$, or $\mu \in E_{\Sigma}$ and $\nu \notin E_{\Sigma}$;

(ii) An infra-tp-soft $T_{1}$-space if for every $\nu \neq \mu \in \mathbb{Y}$, there exist infrasoft open sets $E_{\Sigma}$ and $F_{\Sigma}$ such that $\nu \in E_{\Sigma}, \mu \notin E_{\Sigma}$ and $\mu \in F_{\Sigma}, \nu \notin F_{\Sigma} ;$

(iii) An infra-t $p$-soft $T_{2}$-space (or an infra-tp-soft Hausdorff space) if for every $\nu \neq \mu \in \mathbb{Y}$, there exist 
disjoint infrasoft open sets $E_{\Sigma}$ and $F_{\Sigma}$ such that $\nu \in E_{\Sigma}, \mu \notin E_{\Sigma}$ and $\mu \in F_{\Sigma}, \nu \notin F_{\Sigma} ;$

(iv) An infra-t $p$-soft regular space if for every infrasoft closed set $H_{\Sigma}$ such that $\nu \notin H_{\Sigma}$, there exist disjoint infrasoft open sets $E_{\Sigma}$ and $F_{\Sigma}$ such that $H_{\Sigma} \widetilde{\subseteq} E_{\Sigma}$ and $v \in F_{\Sigma}$;

(v) An infrasoft normal space if disjoint infrasoft closed sets are separated by disjoint infrasoft open sets;

(vi) An infra-t $t p$-soft $T_{3}$ (resp., infra- $t p$-soft $T_{4}$ ) -space if it is infra-t $p$-soft regular (resp., infrasoft normal) and infra-t $p$-soft $T_{1}$.

Before we investigate the relationships between the above infrasoft axioms, we need the following auxiliary result.

Lemma 1. Every infrasoft open (infrasoft closed) subset of an infra-tp-soft regular space is stable; i.e., every infra-tp-soft regular space is stable.

Proof. Let $E_{\Sigma}$ be an infrasoft open subset of an infra-tp-soft regular space $(\mathbb{Y}, \delta, \Sigma)$. Suppose that $E_{\Sigma}$ is unstable; i.e., there exist $\nu \in \mathbb{Y}$ and $\sigma, \sigma \prime \in \sum$ such that $\nu \in E(\sigma)$ and $\nu \notin E(\sigma \prime)$. Now, $E_{\Sigma}^{c}$ is an infrasoft closed set such that $\nu \notin E^{c}(\sigma)$ and $\nu \in E^{c}(\sigma \prime)$. It is clear that for any infrasoft open set $F_{\Sigma}$ containing $E_{\Sigma}^{c}$ we obtain $\nu \Subset F_{\Sigma}$; this implies we cannot find an infrasoft open set whose intersection with $F_{\Sigma}$ is the null soft set such that $v$ totally belongs to it. Hence, $E_{\Sigma}$ must be stable.

One can similarly prove the lemma for an infrasoft closed set.

Corollary 1. The number of infrasoft open subsets of a finite infra-tp-soft regular space $(\mathbb{Y}, \delta, \Sigma)$ is greater than or equal to $2^{|\mathbb{Y}|}$.

Proposition 4. Every infra-tp-soft $T_{i}$-space is an infratp-soft $T_{i-1}$-space for $i=1,2,3$.

Proof. The proof is clear for the cases $i=1,2$. To prove the case of $i=3$, let $\nu, \mu$ be two distinct elements in an infra$t p$-soft $T_{3}$-space. Then there exists an infrasoft open set $E_{\Sigma}$ such that $\nu \in E_{\Sigma}$ and $\mu \notin E_{\Sigma}$. Now, $E_{\Sigma}^{c}$ is infrasoft closed such that $\nu \notin E_{\Sigma}^{c}$. By hypothesis, there exist disjoint infrasoft open sets $U_{\Sigma}$ and $V_{\Sigma}$ such that $E_{\Sigma}^{c} \widetilde{\subseteq} U_{\Sigma}$ and $\nu \in V_{\Sigma}$. It follows from the above lemma that $\mu \notin E_{\Sigma}$. Then $\mu \in U_{\Sigma}$; hence, we obtain the desired result.

Examples below explain that we cannot reverse the above proposition in general.

Example 1. The collections $\delta_{1}=\left\{\Phi, \widetilde{\mathbb{Y}}, E_{1 \Sigma}, E_{3 \Sigma}\right\}$, $\delta_{2}=\left\{\Phi, \widetilde{\mathbb{Y}}, E_{4 \Sigma}, E_{5 \Sigma}, E_{6 \Sigma}\right\}$, and $\delta_{3}=\left\{\Phi, \widetilde{\mathbb{Y}}, E_{1 \Sigma}, E_{2 \Sigma}, E_{3 \Sigma}\right\}$ are three infrasoft topologies on the universal set $\mathbb{Y}=\left\{v_{1}, v_{2}\right\}$ with a set of parameters $\Sigma=\left\{\sigma_{1}, \sigma_{2}\right\}$, where

$$
\begin{aligned}
& E_{1 \Sigma}=\left\{\left(\sigma_{1},\left\{\nu_{1}\right\}\right),\left(\sigma_{2},\left\{\nu_{1}\right\}\right)\right\} ; \\
& E_{2 \Sigma}=\left\{\left(\sigma_{1},\left\{\nu_{2}\right\}\right),\left(\sigma_{2},\left\{\nu_{2}\right\}\right)\right\} ; \\
& E_{3 \Sigma}=\left\{\left(\sigma_{1},\left\{\nu_{2}\right\}\right),\left(\sigma_{2}, \varnothing\right)\right\} ; \\
& E_{4 \Sigma}=\left\{\left(\sigma_{1},\left\{\nu_{1}\right\}\right),\left(\sigma_{2}, \mathbb{Y}\right)\right\} ; \\
& E_{5 \Sigma}=\left\{\left(\sigma_{1},\left\{\nu_{2}\right\}\right),\left(\sigma_{2}, \mathbb{Y}\right)\right\} ; \\
& E_{6 \Sigma}=\left\{\left(\sigma_{1}, \varnothing\right),\left(\sigma_{2}, \mathbb{Y}\right)\right\} ;
\end{aligned}
$$

Obviously, $\left(\mathbb{Y}, \delta_{1}, \Sigma\right),\left(\mathbb{Y}, \delta_{2}, \Sigma\right)$, and $\left(\mathbb{Y}, \delta_{3}, \Sigma\right)$ are, respectively, infra-t $p$-soft $T_{0}$, infra-t $p$-soft $T_{1}$, and infra$t p$-soft $T_{2}$. On the other hand, $\left(\mathbb{Y}, \delta_{1}, \Sigma\right)$ is not infra-t $t p$-soft $T_{1},\left(\mathbb{Y}, \delta_{2}, \Sigma\right)$ is not infra-t $p$-soft $T_{2}$, and $\left(\mathbb{Y}, \delta_{3}, \Sigma\right)$ is not infra-tp-soft $T_{3}$.

Remark 1. In the above example, $\left(\mathbb{Y}, \delta_{3}, \Sigma\right)$ is an infra$t p$-soft $T_{4}$-space. As we know, the soft topology and general topology (infrasoft topology and general infratopology) are identical if a set of parameters is a singleton. Then we can say that there is an infrasoft topology which is infra-t $p$-soft $T_{3}$, but not infra-t $p$-soft $T_{4}$. Hence, the concepts of infra- $t p$-soft $T_{3}$ and infra-t $p$-soft $T_{4}$-spaces are independent of each other.

Now, we explore and discuss some properties of infratp-soft $T_{i}$-spaces.

Proposition 5. If $P_{\sigma}^{\nu}$ is an infrasoft closed subset of $(\mathbb{Y}, \delta, \Sigma)$ for each $\nu \in \mathbb{Y}$, then $(\mathbb{Y}, \delta, \Sigma)$ is an infra-tp-soft $T_{1}$-space.

Proof. Let $\nu \neq \mu \in \mathbb{Y}$. By assumption, $\left(P_{\sigma}^{\nu}\right)^{c}$ and $\left(P_{\sigma}^{\mu}\right)^{c}$ are infrasoft open sets. Obviously, $\mu \in\left(P_{\sigma}^{\nu}\right)^{c}, \nu \notin\left(P_{\sigma}^{\nu}\right)^{c}$, and $\nu \in\left(P_{\sigma}^{\mu}\right)^{c}, \mu \notin\left(P_{\sigma}^{\mu}\right)^{c}$. Hence, $(\mathbb{Y}, \delta, \Sigma)$ is infra-tp-soft $T_{1}$.

In Example $1,\left(\mathbb{Y}, \delta_{2}, \Sigma\right)$ is infra-t $p$-soft $T_{1}$. However, $P_{\sigma_{2}}^{v_{1}}$ and $P_{\sigma_{2}}^{v_{2}}$ are not infrasoft closed sets. So, the converse of the above proposition need not be true in general.

Proposition 6. $(\mathbb{Y}, \delta, \Sigma)$ is an infra-tp-soft $T_{2}$-space iff for each $\nu \neq \mu$, there exists an infrasoft open set $E_{\Sigma}$ such that $\nu \in E_{\Sigma}$ and $\mu \notin C l_{\delta}\left(E_{\Sigma}\right)$, where $\mu \notin C l_{\delta}\left(E_{\Sigma}\right)$ if there is an infrasoft open set $G_{\Sigma}$ such that $\mu \in G_{\Sigma}$ and $E_{\Sigma} \widetilde{\cap} G_{\Sigma}=\Phi$.

Proof. Let $v \neq \mu$ in an infra-tp-soft $T_{2}$-space. Then there exist disjoint infrasoft open sets $E_{\Sigma}$ and $F_{\Sigma}$ such that $\nu \in E_{\Sigma}$ and $\mu \in F_{\Sigma}$. Now, $E_{\Sigma} \widetilde{\subseteq} F_{\Sigma}^{c}$ which means $\mathrm{Cl}_{\delta}\left(E_{\Sigma}\right) \widetilde{\subseteq} F_{\Sigma}^{c}$. Since $\mu \notin F^{c}(\sigma)$ for each $\sigma \in \Sigma, \mu \notin \mathrm{Cl}_{\delta}\left(E_{\Sigma}\right)$. Conversely, let $\nu \neq \mu \in \mathbb{Y}$ and $E_{\Sigma}$ be an infrasoft open set such that $\nu \in E_{\Sigma}$ and $\mu \notin \mathrm{Cl}_{\delta}\left(E_{\Sigma}\right)$. Then there is an infrasoft open set $G_{\Sigma}$ such that $\mu \in G_{\Sigma}$ and $E_{\Sigma} \widetilde{\cap} G_{\Sigma}=\Phi$. This ends the proof that $(\mathbb{Y}, \delta, \Sigma)$ is infra-tp-soft $T_{2}$.

Proposition 7. If $(\mathbb{Y}, \delta, \Sigma)$ is an infra-tp-soft regular space, then for each $\nu \in \mathbb{Y}$ and infrasoft open set $H_{\Sigma}$ partially containing $\nu$, there exists an infrasoft open set $G_{\Sigma}$ such that $\nu \in G_{\Sigma} \widetilde{\subseteq} C l_{\delta}\left(G_{\Sigma}\right) \widetilde{\subseteq} H_{\Sigma}$.

Proof. Let $H_{\Sigma}$ be an infrasoft open set partially containing $\nu$. Then $H_{\Sigma}^{c}$ is an infrasoft closed set and $v \notin H_{\Sigma}^{c}$. By 
assumption, there are disjoint infrasoft open sets $E_{\Sigma}$ and $G_{\Sigma}$ such that $H_{\Sigma}^{c} \widetilde{\subseteq} E_{\Sigma}$ and $v \in G_{\Sigma}$. Obviously, $G_{\Sigma} \widetilde{\subseteq} E_{\Sigma}^{c} \widetilde{\subseteq} H_{\Sigma}$. Thus, $\mathrm{Cl}_{\delta}\left(G_{\Sigma}\right) \subseteq E_{\Sigma}^{c} \widetilde{\subseteq} H_{\Sigma}$.

Theorem 3. If $(\mathbb{Y}, \delta, \Sigma)$ is an infra-tp-soft regular space, then the following concepts are identical.
(i) $(\mathbb{Y}, \delta, \Sigma)$ is infra-tp-soft $T_{2}$.
(ii) $(\mathbb{Y}, \delta, \Sigma)$ is infra-tp-soft $T_{1}$.
(iii) $(\mathbb{Y}, \delta, \Sigma)$ is infra-tp-soft $T_{0}$.

Proof. The implications (i) $\longrightarrow$ (ii) $\longrightarrow$ (iii) follow from Proposition 4.

To prove (iii) $\longrightarrow$ (i), let $\nu \neq \mu \in \mathbb{Y}$. Since $(\mathbb{Y}, \delta, \Sigma)$ is infra-tp-soft $T_{0}$, then we have an infrasoft open set $E_{\Sigma}$ such that $\nu \in E_{\Sigma}$ and $\mu \notin E_{\Sigma}$, or $\mu \in E_{\Sigma}$ and $\nu \notin E_{\Sigma}$. Say, $\nu \in E_{\Sigma}$ and $\mu \notin E_{\Sigma}$. It is clear that $\nu \notin E_{\Sigma}^{c}$ and $\mu \in E_{\Sigma}^{c}$. Since $(\mathbb{Y}, \delta, \Sigma)$ is infra-t $t p$-soft regular, there exist two disjoint infrasoft open sets $U_{\Sigma}$ and $V_{\Sigma}$ such that $\nu \in U_{\Sigma}$ and $\mu \in E_{\Sigma}^{c} \widetilde{\subseteq} V_{\Sigma}$. Since $U_{\Sigma}$ and $V_{\Sigma}$ are disjoint, $\mu \notin U_{\Sigma}$ and $\nu \notin V_{\Sigma}$. This ends the proof that $(\mathbb{Y}, \delta, \Sigma)$ is infra-tp-soft $T_{2}$.

Proposition 8. A finite infra-tp-soft $T_{2}$-space $(\mathbb{Y}, \delta, \Sigma)$ contains at least $|\mathbb{Y}|+2$ infrasoft open sets, where $|\mathbb{Y}|>1$.

Proof. We prove the proposition using mathematical induction. When $|\mathbb{Y}|=2$, we need two infrasoft open sets beside the null and absolute soft sets. Then the claim is true for $i=2$. Suppose that the claim is true for $|\mathbb{Y}|=n$; i.e., $|\delta|=n+2$. Without loss of generality, consider $(\mathbb{Y}, \delta, \Sigma)$ as the smallest infra-t $p$-soft $T_{2}$-space. Now, let $|\mathbb{Y}|=n+1$; i.e., $\mathbb{Y}_{\prime}=\mathbb{Y} \cup\{\xi\}$, where $\xi \notin \mathbb{Y}$. Then $\delta \prime=\delta \cup\{\xi\}$ is the smallest infra-tp-soft $T_{2}$-space on $\mathbb{Y}^{\prime}$. Hence, we obtain the desired result.

In the following result we will study the properties and relationships between infrasoft topology and its parametric infratopologies.

Proposition 9. If $(\mathbb{Y}, \delta, \Sigma)$ is an infra-tp-soft $T_{i}$-space, then $\left(\mathbb{Y}, \delta_{\sigma}\right)$ is infra- $T_{i}$ for each $\sigma \in \Sigma$, where $i=2,3$.

Proof. Let $\nu \neq \mu$ in an infra-tp-soft $T_{2}$-space $(\mathbb{Y}, \delta, \Sigma)$. Then there exist disjoint infrasoft open sets $E_{\Sigma}$ and $F_{\Sigma}$ totally containing $\nu$ and $\mu$, respectively. Therefore, $E(\sigma)$ and $F(\sigma)$ are disjoint infraopen sets containing $\nu$ and $\mu$, respectively. Hence, $\left(\mathbb{Y}, \delta_{\sigma}\right)$ is an infra- $T_{2}$-space.

To prove the proposition in case of $i=3$, it suffices to prove the axiom of infraregularity. To do that, let $G$ be an infraclosed subset of $\left(\mathbb{Y}, \delta_{\sigma}\right)$ such that $\nu \notin G$. Since $\delta$ is infrasoft $t p$-regular, a soft set $H_{\Sigma}=\{(\sigma, G)$ : for each $\sigma \in \Sigma\}$ is an infrasoft closed subset of $(\mathbb{Y}, \delta, \Sigma)$ such that $\nu \notin H_{\Sigma}$. By assumption, there exist disjoint infrasoft open sets $E_{\Sigma}$ and $F_{\Sigma}$ such that $H_{\Sigma} \tilde{\subseteq} E_{\Sigma}$ and $\nu \in F_{\Sigma}$. Thus, $E(\sigma)$ and $F(\sigma)$ are disjoint infraopen subsets of $\left(\mathbb{Y}, \delta_{\sigma}\right)$ containing $G$ and $\nu$, respectively. Hence, $\left(\mathbb{Y}, \delta_{\sigma}\right)$ is infraregular, as required.

To demonstrate that the converse of the proposition above fails, we supply the next example.
Example 2. Consider $\mathbb{Y}$ and $\Sigma$ as the same as in Example 1. Then $\delta=\left\{\Phi, \widetilde{\mathbb{Y}}, E_{\Sigma}, F_{\Sigma}\right\}$ is an infrasoft topology on $\mathbb{Y}$, where

$$
\begin{aligned}
& E_{\Sigma}=\left\{\left(\sigma_{1},\{\nu\}\right),\left(\sigma_{2},\{\mu\}\right)\right\}, \\
& F_{\Sigma}=\left\{\left(\sigma_{1},\{\mu\}\right),\left(\sigma_{2},\{\nu\}\right)\right\} .
\end{aligned}
$$

It is clear that $(\mathbb{Y}, \delta, \Sigma)$ is not an infra-tp-soft $T_{0}$-space. On the other hand, $\left(\mathbb{Y}, \delta_{\sigma_{1}}\right)$ and $\left(\mathbb{Y}, \delta_{\sigma_{2}}\right)$ are infra- $T_{3}$ and infra- $T_{4}$.

From the above example and the next two examples, we elucidate that there is not a relationship between infra$t p$-soft $T_{i}$-spaces and their parametric infratopological spaces when $i=0,1,4$.

Example 3. In Example 1 , note that $\left(\mathbb{Y}, \delta_{2_{\sigma}}\right)$ is not infra- $T_{0}$ in spite of the fact that $\left(\mathbb{Y}, \delta_{2}, \Sigma\right)$ is infra-t $p$-soft $T_{1}$.

Example 4. The collection $\delta=\left\{\Phi, \widetilde{\mathbb{Y}}, E_{1 \Sigma}, E_{3 \Sigma}, E_{3 \Sigma}, E_{4 \Sigma}\right\}$ is an infrasoft topology on $\mathbb{Y}=\left\{\nu_{1}, \nu_{2}, \nu_{3}\right\}$ with $\Sigma=\left\{\sigma_{1}, \sigma_{2}\right\}$ as a set of parameters, where

$$
\begin{aligned}
& E_{1 \Sigma}=\left\{\left(\sigma_{1},\left\{\nu_{1}\right\}\right),\left(\sigma_{2}, \mathbb{Y}\right)\right\} ; \\
& E_{2 \Sigma}=\left\{\left(\sigma_{1},\left\{\nu_{2}\right\}\right),\left(\sigma_{2}, \mathbb{Y}\right)\right\} ; \\
& E_{3 \Sigma}=\left\{\left(\sigma_{1},\left\{\nu_{3}\right\}\right),\left(\sigma_{2}, \mathbb{Y}\right)\right\}, \\
& E_{4 \Sigma}=\left\{\left(\sigma_{1}, \varnothing\right),\left(\sigma_{2}, \mathbb{Y}\right)\right\} .
\end{aligned}
$$

One can check that $(\mathbb{Y}, \delta, \Sigma)$ is infra-tp-soft $T_{4}$. But $\left(\mathbb{Y}, \delta_{\sigma_{2}}\right)$ is not infra- $T_{4}$.

Theorem 4. Let $(\mathbb{Y}, \delta, \Sigma)$ be stable. Then, $(\mathbb{Y}, \delta, \Sigma)$ is infratp-soft $T_{i}$ iff $\left(\mathbb{Y}, \delta_{\sigma}\right)$ is infra- $T_{i}$ for each $i=0,1,2,3,4$.

Proof. Since $(\mathbb{Y}, \delta, \Sigma)$ is stable, then $E$ is an infraopen subset of $\left(\mathbb{Y}, \delta_{\sigma}\right)$ if and only if $\{(\sigma, E): \sigma \in \Sigma\}$ is an infrasoft open subset of $(\mathbb{Y}, \delta, \Sigma)$. Hence, the desired result is proved.

Remark 2. Any extended infrasoft topological space is not infra-t $p$-soft regular; hence, it is not infra-t $p$-soft $T_{3}$.

Theorem 5. Let $(\mathbb{Y}, \delta, \Sigma)$ be extended. If there exists $\sigma \in \Sigma$ such that $\left(\mathbb{Y}, \delta_{\sigma}\right)$ is infra- $T_{i}$, then $(\mathbb{Y}, \delta, \Sigma)$ is infra-tp-soft $T_{i}$ for $i=0,1$.

Proof. When $i=1$, let $\nu \neq \mu \in \mathbb{Y}$. Since $\left(\mathbb{Y}, \delta_{\sigma}\right)$ is infra- $T_{1}$, there are two infraopen sets $U, V$ in $\delta_{\sigma}$ such that $\nu \in U, V$ and $\mu \in V, U$. Since $\delta$ is extended, there are two infrasoft open sets $G_{\Sigma}, H_{\Sigma}$ in $\delta$ such that

$$
\begin{array}{ll}
G(\sigma)=U \text { and } G\left(\sigma^{\prime}\right)=\mathbb{Y}, & \text { for each } \sigma^{\prime} \neq \sigma, \\
H(\sigma)=V \text { and } G\left(\sigma^{\prime}\right)=\mathbb{Y}, & \text { for each } \sigma^{\prime} \neq \sigma .
\end{array}
$$

Now, $v \in G_{\Sigma}, \quad \mu \notin G_{\Sigma}$, and $\mu \in H_{\Sigma}, \quad v \notin H_{\Sigma}$. Hence, $(X, \delta, \Sigma)$ is infra-tp-soft $T_{1}$.

The case $i=0$ can be proved similarly.

Theorem 6. Let $(\mathbb{Y}, \delta, \Sigma)$ be extended. If all $\left(\mathbb{Y}, \delta_{\sigma}\right)$ are infra$T_{i}$, then $(X, \delta, \Sigma)$ is infra-tp-soft $T_{i}$ for $i=0,1,2,4$. 
Proof. We only prove the theorem when $i=4$.

The case of $i=1$ was proved in the above theorem. So, it suffices to prove the axioms of infrasoft normality. To do this, let $H_{\Sigma}, L_{\Sigma}$ be two disjoint infrasoft closed sets. Then $H(\sigma)$ and $L(\sigma)$ are two disjoint infraclosed sets for each $\sigma \in \Sigma$. Since $\left(X, \delta_{\sigma}\right)$ is infranormal, there are two disjoint infraopen sets $U_{\sigma}$ and $V_{\sigma}$ such that $H(\sigma) \subseteq U_{\sigma}$ and $L(\sigma) \subseteq V_{\sigma}$. Since $(\mathbb{Y}, \delta, \Sigma)$ is extended, there are disjoint infrasoft open subsets $G_{\Sigma}, F_{\Sigma}$ of $(\mathbb{Y}, \delta, \Sigma)$ such that

$$
\begin{array}{ll}
G(\sigma)=U_{\sigma}, & \text { for each } \sigma \in \Sigma, \\
F(\sigma)=V_{\sigma}, & \text { for each } \sigma \in \Sigma .
\end{array}
$$

Thus, $(X, \delta, \Sigma)$ is infrasoft normal. Hence, it is infratp-soft $T_{4}$.

Theorem 7. Every soft subspace $\left(\mathbb{Z}, \delta_{\mathbb{Z}}, \Sigma\right)$ of an infratp-soft $T_{i}$-space $(\mathbb{Y}, \delta, \Sigma)$ is an infra-tp-soft $T_{i}$-space, for $i=0,1,2,3$.

Proof. We give a proof for $i=3$. The other cases are made similarly.

First, we prove that $\left(\mathbb{Z}, \delta_{\mathbb{Z}}, \Sigma\right)$ is infra-tp-soft $T_{1}$. To do this, let $\nu \neq \mu \in \mathbb{Z} \subseteq \mathbb{Y}$. Since $(\mathbb{Y}, \delta, \Sigma)$ is infra-tp-soft $T_{1}, \delta$ contains infrasoft open sets $E_{\Sigma}$ and $F_{\Sigma}$ such that $\nu \in E_{\Sigma}, \mu \notin E_{\Sigma}$ and $\mu \in F_{\Sigma}, v \notin F_{\Sigma}$. Now, $U_{\Sigma}=\widetilde{\mathbb{V} \cap} E_{\Sigma}$ and $V_{\Sigma}=\widetilde{\mathbb{V} \cap} F_{\Sigma}$ are infrasoft open subsets of $\left(\mathbb{Z}, \delta_{\mathbb{Z}}, \Sigma\right)$ such that $\nu \in U_{\Sigma}, \mu \notin U_{\Sigma}$ and $\mu \in V_{\Sigma}, \nu \notin V_{\Sigma}$. Thus, $\left(\mathbb{Z}, \delta_{\mathbb{Z}}, \Sigma\right)$ is infra-tp-soft $T_{1}$.

Second, we prove that $\left(\mathbb{Z}, \delta_{\mathbb{Z}}, \Sigma\right)$ is infra-t $p$-soft regular. To do this, let $L_{\Sigma}$ be an infrasoft closed subset of $\left(\mathbb{Z}, \delta_{\mathbb{Z}}, \Sigma\right)$ and $\mu \in \mathbb{Z}$ such that $\mu \notin L_{\Sigma}$. Then there exists an infrasoft closed subset $H_{\Sigma}$ of $(\mathbb{Y}, \delta, \Sigma)$ such that $L_{\Sigma}=\widetilde{\mathbb{\nabla} \cap} H_{\Sigma}$. By assumption, there exist disjoint infrasoft open sets $E_{\Sigma}$ and $F_{\Sigma}$

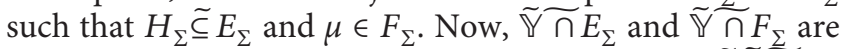
infrasoft open subsets of $\left(\mathbb{Z}, \delta_{\mathbb{Z}}, \Sigma\right)$ such that $L_{\Sigma} \widetilde{\widetilde{\mathbb{V}}} \cap E_{\Sigma}$ and $\mu \in \widetilde{\mathbb{Y} \cap} F_{\Sigma}$. Thus, $\left(\mathbb{Z}, \delta_{\mathbb{Z}}, \Sigma\right)$ is infra-t $p$-soft regular. This ends the proof that $\left(\mathbb{Z}, \delta_{\mathbb{Z}}, \Sigma\right)$ is infra-tp-soft $T_{3}$.

Following similar technique given above, one can prove the following result.

Theorem 8. Every infrasoft closed subspace $\left(\mathbb{Z}, \delta_{\mathbb{Z}}, \Sigma\right)$ of an infra-tp-soft $T_{4}$-space $(\mathbb{Y}, \delta, \Sigma)$ is an infra-tp-soft $T_{4}$-space.

Theorem 9. The concepts of infra-tp-soft $T_{i}$-spaces are preserved under finite product spaces, where $i=0,1,2$.

Proof. We give a proof for the theorem when $j=2$. The remaining two cases follow similar lines.

Without loss of generality, we consider $\left(\mathbb{Y}_{1}, \delta_{1}, \Sigma_{1}\right)$ and $\left(\mathbb{V}_{2}, \delta_{2}, \Sigma_{2}\right)$ as two infra-tp-soft $T_{2}$-spaces. Let $\left(\nu_{1}, \mu_{1}\right) \neq\left(\nu_{2}, \mu_{2}\right)$ in $\mathbb{Y}_{1} \times \mathbb{Y}_{2}$. Then $\nu_{1} \neq \nu_{2}$ or $\mu_{1} \neq \mu_{2}$. Say, $\nu_{1} \neq \nu_{2}$. By assumption, $\delta_{1}$ contains two disjoint infrasoft open sets $E_{\Sigma_{1}}, F_{\Sigma_{1}}$ such that $\nu_{1} \in E_{\Sigma_{1}}$ and $\nu_{2} \notin E_{\Sigma_{1}}$, and $v_{2} \in F_{\Sigma_{1}}$ and $v_{1} \notin F_{\Sigma_{1}}$. Now, $E_{\Sigma_{1}} \times \widetilde{\mathbb{Y}}_{2}$ and $F_{\Sigma_{1}} \times \widetilde{\mathbb{Y}}_{2}$ are disjoint infrasoft open sets such that $\left(\nu_{1}, \mu_{1}\right) \in E_{\Sigma_{1}} \times{\widetilde{\mathbb{Y}_{2}}}_{2}$ and $\left(\nu_{2}, \mu_{2}\right) \notin E_{\Sigma_{1}} \times \widetilde{\mathbb{Y}}_{2}$, and $\quad\left(\nu_{2}, \mu_{2}\right) \in F_{\Sigma_{1}} \times \widetilde{\mathbb{Y}}_{2} \quad$ and $\left(\nu_{1}, \mu_{1}\right) \notin F_{\Sigma_{1}} \times \widetilde{\mathbb{Y}}_{2}$. Hence, $\mathbb{Y}_{1} \times \mathbb{Y}_{2}$ is infra-t $p$-soft $T_{2}$.
We close this section by studying the behaviors of infra$t p$-soft $T_{i}$-spaces under some types of soft maps.

Proposition 10. The inverse image of infra-tp-soft $T_{i}$-spaces under an infrasoft continuous map $f_{\eta}$ is infra-tp-soft $T_{i}$ for $i=0,1,2$ provided that $f$ is injective and $\eta$ is surjective.

Proof. When $i=2$, consider $f_{\eta}:(\mathbb{Y}, \delta, \Sigma) \longrightarrow(\mathbb{Z}, \theta, \Omega)$ is an infrasoft continuous map. Let $\nu \neq \mu \in \mathbb{Y}$. By the injectivity of $f$, there are only two distinct points $\zeta, \xi \in \mathbb{Z}$ such that $f(\nu)=\zeta$ and $f(\mu)=\xi$. By assumption, there are two disjoint infrasoft open sets $E_{\Omega}$ and $G_{\Omega}$ such that $\zeta \in E_{\Omega}, \xi \notin E_{\Omega}$ and $\xi \in G_{\Omega}, \zeta \notin G_{\Omega}$. Now, $f_{\eta}^{-1}\left(E_{\Omega}\right)$ and $f_{\eta}^{-1}\left(G_{\Omega}\right)$ are disjoint infrasoft open sets. By the surjectivity of $\eta$ we obtain from Proposition 1 that $v \in f_{\eta}^{-1}\left(E_{\Omega}\right), \quad \mu \notin f_{\eta}^{-1}\left(E_{\Omega}\right)$ and $\mu \in f_{\eta}^{-1}\left(G_{\Omega}\right), \nu \notin f_{\eta}^{-1}\left(G_{\Omega}\right)$. Hence, we get the coveted result.

In a similar manner, one can prove the next results.

Proposition 11. The inverse image of infra-tp-soft $T_{i}$-spaces under a bijective infrasoft continuous map $f_{\eta}$ is infra-tp-soft $T_{i}$ for $i=0,1,2,3,4$.

Proposition 12. The image of infra-tp-soft $T_{i}$-spaces under a bijective infrasoft open map $f_{\eta}$ is infra-tp-soft $T_{i}$ for $i=0,1,2,3,4$.

Proposition 13. The property of being an infra-tp-soft $T_{i}$-space $(i=0,1,2,3,4)$ is preserved under an infrasoft homeomorphism map.

\section{Infra-t $t$-Soft $T_{i}$-Spaces $(i==0,1,2,3,4)$}

In this part, we apply the relations of total belong and total nonbelong to define the concepts of infra-tt-soft $T_{i}$-spaces $(i=0,1,2,3,4)$, where $t t$ is the abbreviation for the first letter of total belong and total nonbelong relations that are used to define these spaces. We provide some counterexamples to illustrate the relationships between these concepts and the interrelations between them and infra- $t p$-soft $T_{i}$-spaces.

Definition 19. $(\mathbb{Y}, \delta, \Sigma)$ is said to be

(i) An infra-tt-soft $T_{0}$-space if for every $\nu \neq \mu \in \mathbb{Y}$, there is an infrasoft open set $E_{\Sigma}$ such that $\nu \in E_{\Sigma}, \mu \notin E_{\Sigma}$, or $\mu \in E_{\Sigma}, \nu \notin E_{\Sigma}$;

(ii) An infra-tt-soft $T_{1}$-space if for every $\nu \neq \mu \in \mathbb{Y}$, there are infrasoft open sets $E_{\Sigma}$ and $F_{\Sigma}$ such that $\nu \in E_{\Sigma}, F_{\Sigma}$ and $\mu \in F_{\Sigma}, E_{\Sigma}$. In other words, $\nu \in E_{\Sigma}, \mu \notin E_{\Sigma}$ and $\mu \in F_{\Sigma}, \nu \notin F_{\Sigma}$;

(iii) An infra-tt-soft $T_{2}$-space if for every $\nu \neq \mu \in \mathbb{Y}$, there are two disjoint infrasoft open sets $E_{\Sigma}$ and $F_{\Sigma}$ such that $\nu \in E_{\Sigma} \backslash F_{\Sigma}$ and $\mu \in F_{\Sigma} \backslash E_{\Sigma}$;

(iv) An infra-tt-soft regular space if for every infrasoft closed set $H_{\Sigma}$ such that $\nu \notin H_{\Sigma}$, there are disjoint infrasoft open sets $E_{\Sigma}$ and $F_{\Sigma}$ such that $H_{\Sigma} \widetilde{\subseteq} E_{\Sigma}$ and $\nu \in F_{\Sigma}$; 
(v) An infra-tt-soft $T_{3}$ (resp., infra-tt-soft $T_{4}$ ) -space if it is infra-tt-soft regular (resp., infrasoft normal) and infra-tt-soft $T_{1}$.

This section begins with the next result which describes the relationships between infra-t $t$-soft $T_{i}$-spaces as well as the interrelations between them and infra-t $p$-soft $T_{i}$-spaces.

\section{Proposition 14}

(i) Every infra-tt-soft $T_{i}$-space is infra-tt-soft $T_{i-1}$ for $i=1,2,3$.

(ii) The concepts of infra-tt-soft $T_{2}$-space and infratp-soft $T_{2}$-space are identical.

(iii) Every infra-tt-soft $T_{i}$-space is infra-tp-soft $T_{i}$ for $i=0,1,4$.

(iv) Every infra-tp-soft $T_{3}$-space is infra-tt-soft $T_{3}$

Proof

(i) The proof of the cases $i=1,2$ follows directly from the above definition. To prove case $i=3$, let $\nu, \mu$ be two distinct elements in an infra-tt-soft $T_{3}$-space. Then there exists an infrasoft open set $E_{\Sigma}$ such that $\nu \in E_{\Sigma}$ and $\mu \notin E_{\Sigma}$. Now, $E_{\Sigma}^{c}$ is infrasoft closed such that $v \notin E_{\Sigma}^{c}$. By hypothesis, there exist disjoint infrasoft open sets $U_{\Sigma}$ and $V_{\Sigma}$ such that $E_{\Sigma}^{c} \widetilde{\subseteq} U_{\Sigma}$ and $\nu \in V_{\Sigma}$. Obviously, $\mu \in U_{\Sigma}$; hence, we obtain the desired result.

(ii) Let $E_{\Sigma}$ and $F_{\Sigma}$ be two disjoint soft sets such that $\nu \in E_{\Sigma}$ and $\mu \in F_{\Sigma}$. Then $\mu \notin E_{\Sigma}$ iff $\mu \notin E_{\Sigma}$, and $\nu \notin F_{\Sigma}$ iff $\nu \notin F_{\Sigma}$. This proves the required equivalence.

(iii) obvious.

(iv) It is clear that every infra-tp-soft regular space is infra- $t$-soft regular. According to Lemma 1, the concepts of infra-tt-soft $T_{1}$-space and infra-t $p$-soft $T_{1}$-space are identical. Hence, we get the coveted result.

To elucidate that the converses of the above proposition fail, we provide the next three counterexamples.

Example 5. Let $\mathbb{Y}$ be any infinite set and $\nu, \mu \in \mathbb{Y}$. We define the following three infrasoft topologies on $\mathbb{Y}$ with $\Sigma$ as set of parameters such that $|\Sigma| \neq 1$.

$$
\begin{aligned}
& \delta_{1}=\{\widetilde{\mathbb{Y}}\} \cup\left\{E_{\Sigma} \tilde{\subseteq} \widetilde{\mathbb{V}}: \nu \notin E_{\Sigma}\right\} ; \\
& \delta_{2}=\{\Phi\} \cup\left\{E_{\Sigma} \widetilde{\subseteq} \widetilde{\mathbb{Y}}: E_{\Sigma}^{c} \text { is finite }\right\}, \\
& \delta_{3}=\{\widetilde{\mathbb{Y}}\} \cup\left\{E_{\Sigma} \widetilde{\subseteq} \widetilde{\mathbb{V}}: \nu \notin E_{\Sigma} \text { or } \mu \notin E_{\Sigma}\right\} .
\end{aligned}
$$

Now, we can note that $\left(\mathbb{Y}, \delta_{1}, \Sigma\right),\left(\mathbb{Y}, \delta_{2}, \Sigma\right)$, and $\left(\mathbb{Y}, \delta_{3}, \Sigma\right)$ are, respectively, infra-tt-soft $T_{0}$, infra-tt-soft $T_{1}$, and infra-tt-soft $T_{2}$. On the other hand, we can check that $\left(\mathbb{Y}, \delta_{1}, \Sigma\right)$ is not infra-tt-soft $T_{1}$ and $\left(\mathbb{Y}, \delta_{2}, \Sigma\right)$ is not infra$t t$-soft $T_{2}$. Also, $\left(\mathbb{Y}, \delta_{3}, \Sigma\right)$ is not infra-tt-soft $T_{3}$ because there does not exist an infrasoft open set (except for the absolute soft set) containing an infrasoft closed set $E_{\Sigma}=\{(\sigma,\{\nu, \mu\})$ : for each $\sigma \in \Sigma\}$.
Example 6. Consider $(\mathbb{Y}, \delta, \Sigma)$ as the same as given in $\mathrm{Ex}$ ample 4 . Then $(\mathbb{Y}, \delta, \Sigma)$ is an infra-tp-soft $T_{4}$-space but it is not infra-tt-soft $T_{0}$.

Example 7. Any discrete soft topological space is infra$t t$-soft $T_{3}$. On the other hand, it contains some infrasoft open sets which are not stable, so it is not infra-t $p$-soft regular. Hence, it is not infra-tp-soft $T_{3}$.

Remark 3. We show that $\left(\mathbb{Y}, \delta_{3}, \Sigma\right)$ given in Example 5 is not infra- $t$-soft $T_{3}$. In contrast, one can check that it is infra$t t$-soft $T_{4}$. According to Remark 1 there is an infra- $t$-soft $T_{3}$-space which is not infra-tt-soft $T_{4}$. Hence, the concepts of infra-tt-soft $T_{3}$ and infra-tt-soft $T_{4}$-spaces are independent of each other.

Theorem 10. Let $(\mathbb{Y}, \delta, \Sigma)$ be a stable space. Then the following statements hold.

(i) $(\mathbb{Y}, \delta, \Sigma)$ is infra-tt-soft $T_{0} \Leftrightarrow(\mathbb{Y}, \delta, \Sigma)$ is infra-tp-soft $T_{0}$.

(ii) $(\mathbb{Y}, \delta, \Sigma)$ is infra-tt-soft $T_{1} \Leftrightarrow(\mathbb{Y}, \delta, \Sigma)$ is infra-tp-soft $T_{1}$.

(iii) $(\mathbb{Y}, \delta, \Sigma)$ is infra-tt-soft $T_{2} \Leftrightarrow(\mathbb{Y}, \delta, \Sigma)$ is infra-tp-soft $T_{2}$.

(iv) $(\mathbb{Y}, \delta, \Sigma)$ is infra-tt-soft $T_{3} \Leftrightarrow(\mathbb{Y}, \delta, \Sigma)$ is infra-tp-soft $T_{3}$.

(v) $(\mathbb{Y}, \delta, \Sigma)$ is infra-tt-soft $T_{4} \Leftrightarrow(\mathbb{Y}, \delta, \Sigma)$ is infra-tp-soft $T_{4}$.

Proof. It comes from the fact that partial nonbelong and total nonbelong relations are identical with respect to the stable soft sets.

In what follows, we study and investigate some properties of infra-tt-soft $T_{i}$-spaces.

Proposition 15. If every singleton soft subset of $(\mathbb{Y}, \delta, \Sigma)$ is infrasoft closed, then $(\mathbb{Y}, \delta, \Sigma)$ is an infra-tt-soft $T_{1}$-space.

Proof. Let $\nu \neq \mu \in \mathbb{Y}$. By assumption, $\left(\nu_{\Sigma}\right)^{c}$ and $\left(\mu_{\Sigma}\right)^{c}$ are infrasoft open sets. Obviously, $\mu \in\left(\nu_{\Sigma}\right)^{c}, \nu \notin\left(\nu_{\Sigma}\right)^{c}$, and $\nu \in\left(\mu_{\Sigma}\right)^{c}, \mu \notin\left(\mu_{\Sigma}\right)^{c}$. Hence, $(\mathbb{Y}, \delta, \Sigma)$ is infra-tt-soft $T_{1}$.

The converse of the above proposition is not always true as the next counterexample explains.

Example 8. Let $\delta=\{\widetilde{\mathbb{N}}\} \cup\left\{F_{\Sigma} \widetilde{\widetilde{E}} \widetilde{\mathbb{N}}: F_{\Sigma}\right.$ is finite $\}$ be an infrasoft topology on the set of natural numbers $\mathbb{N}$ with $\Sigma$ as any set of parameters. It is clear that $(\mathbb{N}, \delta, \Sigma)$ is infra-tt-soft $T_{1}$. But every singleton soft set is not infrasoft closed.

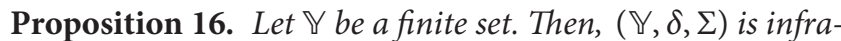
tt-soft $T_{1}$ iff every singleton soft set is infrasoft open.

Proof. To prove the necessary condition, let $\nu_{\Sigma}$ be a singleton soft set. Then for each $\mu_{i} \neq v \in \mathbb{Y}$ there exists an infrasoft open set $E_{\Sigma}$ such that $\nu \in E_{i \Sigma}$ and $\mu_{i} \notin E_{i \Sigma}$. Now, 
$\widetilde{\cap}_{i=1}^{n-1} E_{i \Sigma}=\nu_{\Sigma}$ is an infrasoft open set, where $n$ is the cardinality number of $\mathbb{Y}$.

The sufficient condition is obvious.

Corollary 2. If $(\mathbb{Y}, \delta, \Sigma)$ is a finite infra-tt-soft $T_{1}$-space, then $|\delta| \geq|\bigvee|+2$.

Corollary 3. If $(\mathbb{Y}, \delta, \Sigma)$ is an infra-tt-soft $T_{1}$-space, then $\nu_{\Sigma}=\widetilde{\cap}\left\{E_{\Sigma}: \nu \in E_{\Sigma} \in \delta\right\}$ for all $\nu \in \mathbb{Y}$.

Proposition 17. $(\mathbb{Y}, \delta, \Sigma)$ is an infra-tt-soft $T_{2}$-space iff for every $\nu \neq \mu$, there exists an infrasoft open set $E_{\Sigma}$ such that $\nu \in E_{\Sigma}$ and $\mu \notin C l_{\delta}\left(E_{\Sigma}\right)$.

Proof. It comes from Proposition 6 and item (ii) of Proposition 14.

Proposition 18. A finite infra-tp-soft $T_{2}$-space $(\mathbb{Y}, \delta, \Sigma)$ contains at least $|\mathbb{Y}|+2$ infrasoft open sets, where $|\mathbb{Y}|>1$.

Proof. It comes from Proposition 8 and item (ii) of Proposition 14.

Proposition 19. If $(\mathbb{Y}, \delta, \Sigma)$ is an infra-tt-soft regular space, then for each $\nu \in \mathbb{Y}$ and infrasoft open set $H_{\Sigma}$ totally containing $\nu$, there exists an infrasoft open set $G_{\Sigma}$ such that $\nu \in G_{\Sigma} \widetilde{\subseteq} C l_{\delta}\left(G_{\Sigma}\right) \widetilde{\subseteq} H_{\Sigma}$.

Proof. It follows similar arguments given in the proof of Proposition 7.

Theorem 11. Let $(\mathbb{Y}, \delta, \Sigma)$ be infra-tp-soft regular. Then $(\mathbb{Y}, \delta, \Sigma)$ is infra-tt-soft $T_{2}$ iff it is infra-tp-soft $T_{0}$.

Proof. It comes from Theorem 3 and item (ii) of Proposition 14.

Corollary 4. The following concepts are equivalent under an infra-tp-soft regular space $(\mathbb{Y}, \delta, \Sigma)$.

(i) $(\mathbb{Y}, \delta, \Sigma)$ is infra-tt-soft $T_{2}$;

(ii) $(\mathbb{Y}, \delta, \Sigma)$ is infra-tt-soft $T_{1}$;

(iii) $(\mathbb{Y}, \delta, \Sigma)$ is infra-tt-soft $T_{0}$;

(iv) $(\mathbb{Y}, \delta, \Sigma)$ is infra-tp-soft $T_{0}$;

(v) $(\mathbb{Y}, \delta, \Sigma)$ is infra-tp-soft $T_{1}$.

Now, we determine the conditions under which the property infra-tt-soft $T_{i}$-spaces is transmitted from infrasoft topology to crisp infratopology and vice versa.

Proposition 20. If $(\mathbb{Y}, \delta, \Sigma)$ is an infra-tt-soft $T_{i}$-space, then $\left(\mathbb{Y}, \delta_{\sigma}\right)$ is infra- $T_{i}$ for each $\sigma \in \Sigma$, where $i=0,1,2$.

Proof. Let $\nu \neq \mu$ in an infra-tt-soft $T_{0}$-space $(\mathbb{Y}, \delta, \Sigma)$. Without loss of generality, there is an infrasoft open set $E_{\Sigma}$ such that $\nu \in E_{\Sigma}$ and $\mu \notin E_{\Sigma}$. Therefore, $E(\sigma)$ is an infraopen set such that $\nu \in E(\sigma)$ and $\mu \notin E(\sigma)$. Hence, $\left(\mathbb{Y}, \delta_{\sigma}\right)$ is an infra- $T_{0}$-space.
The cases $i=1,2$ are achieved similarly.

Example 2 illustrates that the converse of the above proposition fails.

The next remark and example elucidate that there is not a relationship between infra-tt-soft $T_{i}$-spaces and their parametric infratopological spaces when $i=3,4$.

Remark 4. ( $\left.\mathbb{Y}, \delta_{3}, \Sigma\right)$ given in Example 5 is not infra-tt-soft $T_{3}$ but all its parametric infratopological spaces are infra- $T_{3}$. Also, $\left(\mathbb{Y}, \delta_{1}, \Sigma\right)$ given in Example 5 is not infra- $t$-soft $T_{4}$ but all its parametric infratopological spaces are infra- $T_{4}$.

Example 9. Let $\mathbb{Y}=\left\{v_{1}, v_{2}\right\}$ be the universal set and $\Sigma=$ $\left\{\sigma_{1}, \sigma_{2}\right\}$ be a parameters set. We define the following soft sets $E_{i \Sigma}$ over $\mathbb{Y}$ as follows:

$$
\begin{aligned}
& E_{1 \Sigma}=\left\{\left(\sigma_{1},\left\{v_{1}\right\}\right),\left(\sigma_{2}, \mathbb{Y}\right)\right\} ; \\
& E_{2 \Sigma}=\left\{\left(\sigma_{1},\left\{v_{2}\right\}\right),\left(\sigma_{2}, \mathbb{Y}\right)\right\}, \\
& E_{3 \Sigma}=\left\{\left(\sigma_{1}, \varnothing\right),\left(\sigma_{2}, \mathbb{Y}\right)\right\} .
\end{aligned}
$$

Then $\delta=\left\{\Phi, \widetilde{\mathbb{Y}}, E_{1 \Sigma}, E_{2 \Sigma}, E_{3 \Sigma}\right\}$ is an infrasoft topology on Y. Obviously, $(\mathbb{Y}, \delta, \Sigma)$ is not infra-tt-soft $T_{0}$. But its parametric infratopological space $\left(\mathbb{Y}, \delta_{\sigma_{1}}\right)$ is infra- $T_{4}$ and infra- $T_{3}$.

Theorem 12. Let $(\mathbb{Y}, \delta, \Sigma)$ be stable (infra-tp-soft regular). Then, $\left(\mathbb{Y}, \delta_{\sigma}\right)$ is infra- $T_{i}$ iff $(\mathbb{Y}, \delta, \Sigma)$ is infra-tt-soft $T_{i}$ for each $i=0,1,2,3,4$.

Proof. It is straightforward.

Theorem 13. Let $(\mathbb{Y}, \delta, \Sigma)$ be extended. If all $\left(\mathbb{Y}, \delta_{\sigma}\right)$ are infra- $T_{i}$, then $(X, \delta, \Sigma)$ is infra-tt-soft $T_{i}$ for $i=0,1,2,3,4$.

Proof. The cases of $i=0,1,2$ were proved in Theorem 1 . The case of $i=4$ can be made similar to the proof of Theorem 6 . It remains to prove case $i=3$. It suffices to prove the axiom of infra-tt-soft regularity. To do this, let $H_{\Sigma}$ be an infrasoft closed set such that $v \notin H_{\Sigma}$. Then $\nu \notin H(\sigma)$ for each $\sigma \in \Sigma$. Since $H(\sigma)$ is an infraclosed set and $\left(\mathbb{Y}, \delta_{\sigma}\right)$ is infraregular, there exist disjoint infraopen sets $U_{\sigma}$ and $V_{\sigma}$ such that $H(\sigma) \subseteq U_{\sigma}$ and $\nu \in V_{\sigma}$. Since $(\mathbb{Y}, \delta, \Sigma)$ is extended, then there exist infrasoft open sets $G_{\Sigma}$ and $F_{\Sigma}$ such that $G(\sigma)=U_{\sigma}$ and $F(\sigma)=V_{\sigma}$ for each $\sigma \in \Sigma$. Obviously, $H_{\Sigma} \widetilde{\subseteq} G_{\Sigma}$ and $v \in F_{\Sigma}$. The disjointness of $G_{\Sigma}$ and $F_{\Sigma}$ proves that $(\mathbb{Y}, \delta, \Sigma)$ is infratt-soft regular.

The following findings can be proved in a similar way to their counterparts in Section 3.

Theorem 14. Every soft subspace $\left(\mathbb{Z}, \delta_{\mathbb{Z}}, \Sigma\right)$ of an infratt-soft $T_{i}$-space $(\mathbb{Y}, \delta, \Sigma)$ is an infra-tt-soft $T_{i}$-space for $i=0,1,2,3$.

Theorem 15. Every infrasoft closed subspace $\left(\mathbb{Z}, \delta_{\mathbb{Z}}, \Sigma\right)$ of an infra-tt-soft $T_{4}$-space $(\mathbb{Y}, \delta, \Sigma)$ is an infra-tt-soft $T_{4}$-space.

Theorem 16. The concepts of infra-tt-soft $T_{i}$-spaces are preserved under finite product spaces, where $i=0,1,2$. 


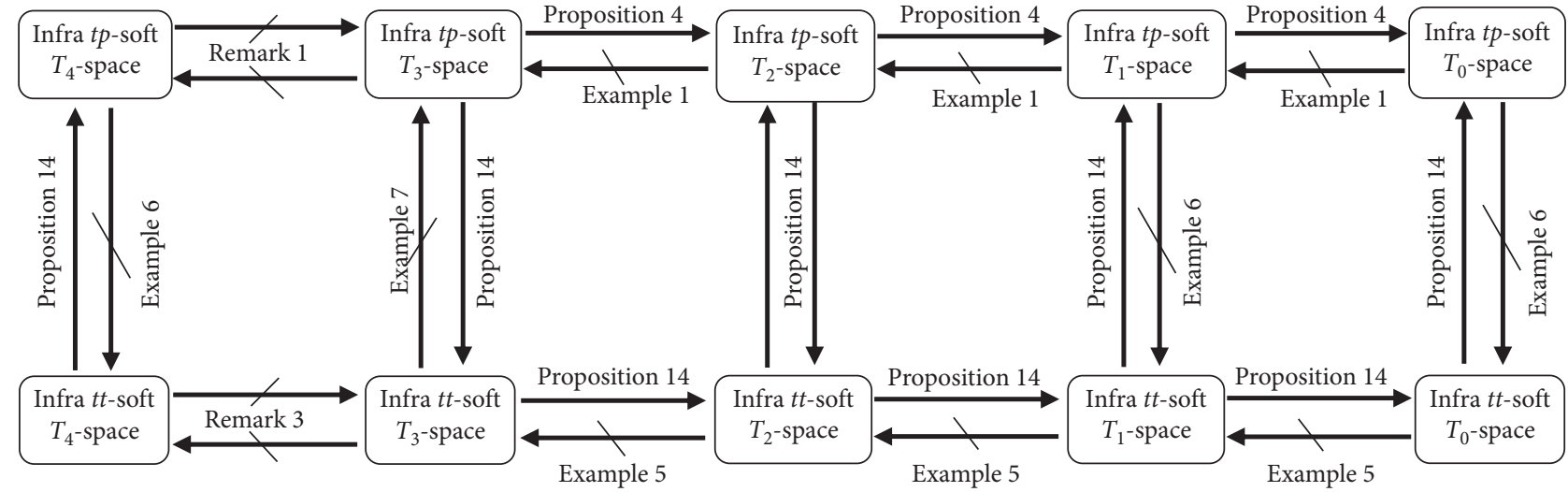

Figure 1: The relationships between infrasoft separation axioms.

Proposition 21. The inverse image of infra-tt-soft $T_{i}$-spaces under an infrasoft continuous map $f_{\eta}$ is infra-tt-soft $T_{i}$ for $i=0,1,2$ provided that $f$ is injective.

Proposition 22. The inverse image of infra-tt-soft $T_{i}$-spaces under a bijective infrasoft continuous map $f_{\eta}$ is infra-tt-soft $T_{i}$ for $i=0,1,2,3,4$.

Proposition 23. The image of infra-tt-soft $T_{i}$-spaces under a bijective infrasoft open map $f_{\eta}$ is infra-tt-soft $T_{i}$ for $i=0,1,2,3,4$.

Proposition 24. The property of being an infra-tt-soft $T_{i}$-space $(i=0,1,2,3,4)$ is preserved under an infrasoft homeomorphism map.

In the end of this work, we summarize the relationships between the two introduced infrasoft separation axioms in Figure 1.

\section{Conclusion and Future Work}

In this paper, we have introduced two types of separation axioms in the frame of infrasoft topological spaces with respect to distinct ordinary points. The reasons beyond this study are the following.

(1) Construct new classes of soft spaces and explore the (classical) topological properties that are valid in these spaces.

(2) Investigate these soft spaces in the frame of rough sets models as those given [34-36] and show whether they have an influence on the rough approximations and accuracy measures.

(3) Obtain new results induced from the interaction between these soft spaces and some celebrated concepts such as infrasoft connectedness and infrasoft compactness which we will introduce later.

We outline the achievements of this work in the following.
(1) Formulate the concepts of infra-t $t p$-soft $T_{i}$-spaces for $i=0,1,2,3,4$ using total belong and partial nonbelong relations.

(2) Formulate the concepts of infra-tt-soft $T_{i}$-spaces for $i=0,1,2,3,4$ using total belong and total nonbelong relations.

(3) Provide some counterexamples to show the relationships between the spaces in each type of them as well as the interrelationships between the two types.

(4) Study the transmission property of these two types of soft spaces between infrasoft topology and its parametric infratopologies.

(5) Discuss the image and preimage of these two types of soft spaces under some infrasoft maps.

In upcoming papers, we plan to do the following.

(1) Establish novel types of soft spaces using the two pairs of relations $(\Subset, \notin)$ and $(\Subset, \notin)$.

(2) Define the celebrated generalizations of open sets in the context of infrasoft topological spaces such as infrasoft $\alpha$-open, infrasoft semiopen, infrasoft preopen, infrasoft $b$-open, and infrasoft $\beta$-open sets.

(3) Carry out further investigations in the areas of rough set theory and infrasoft topologies.

\section{Data Availability}

No data were used to support this study.

\section{Conflicts of Interest}

The authors declare no conflicts of interest.

\section{References}

[1] D. Molodtsov, "Soft set theory-first results," Computers \& Mathematics with Applications, vol. 37, pp. 19-31, 1999.

[2] P. K. Maji, R. Biswas, and R. Roy, "Soft set theory," Computers \& Mathematics with Applications, vol. 45, pp. 555-562, 2003.

[3] P. K. Maji, R. Biswas, and R. Roy, "An application of soft sets in a decision making problem," Computers \& Mathematics with Applications, vol. 44, pp. 1077-1083, 2002. 
[4] M. I. Ali, F. Feng, X. Liu, W. K. Min, and M. Shabir, "On some new operations in soft set theory," Computers \& Mathematics with Applications, vol. 57, no. 9, pp. 1547-1553, 2009.

[5] K. Qin and Z. Hong, "On soft equality," Journal of Computational and Applied Mathematics, vol. 234, pp. 1347-1355, 2010.

[6] M. Abbas, M. Ali, and S. Romaguera, "Generalized operations in soft set theory via relaxed conditions on parameters," Filomat, vol. 31, no. 19, pp. 5955-5964, 2017.

[7] T. M. Al-shami and M. E. El-Shafei, "T-soft equality relation," Turkish Journal of Mathematics, vol. 44, no. 4, pp. 1427-1441, 2020.

[8] N. Cağman and S. Enginoğlu, "Soft matrix theory and its decision making," Computers and Mathematics with Applications, vol. 59, pp. 3308-3314, 2010.

[9] T. M. Al-shami and M. E. El-Shafei, "Partial belong relation on soft separation axioms and decision-making problem, two birds with one stone," Soft Computing, vol. 24, no. 7, pp. 5377-5387, 2020.

[10] M. E. El-Shafei and T. M. Al-shami, "Applications of partial belong and total non-belong relations on soft separation axioms and decision-making problem," Computational and Applied Mathematics, vol. 39, no. 3, 2020.

[11] M. K. El-Bably and A. El Atik, "Soft $\beta$-rough sets and their application to determine COVID-19," Turkish Journal of Mathematics, vol. 45, no. 3, pp. 1133-1148, 2021.

[12] M. El Sayed, A. A. Q. Al Qubati, and M. K. El-Bably, "Soft prerough sets and its applications in decision making," Mathematical Biosciences and Engineering, vol. 17, no. 5, pp. 6045-6063, 2020.

[13] M. Shabir and M. Naz, "On soft topological spaces," Computers and Mathematics with Applications, vol. 61, pp. 1786-1799, 2011.

[14] J. C. R. Alcantud, "Soft open bases and a novel construction of soft topologies from bases for topologies," Mathematics, vol. 8 , no. 5 , p. $672,2020$.

[15] J. C. R. Alcantud, "An operational characterization of soft topologies by crisp topologies," Mathematics, vol. 9, no. 14, p. $1656,2021$.

[16] A. Aygünoğlu and H. Aygün, "Some notes on soft topological spaces," Neural Computing and Applications, vol. 21, pp. 113-119, 2012.

[17] L. D. R. Kočinac, T. M. Al-shami, and V. Çetkin, "Selection principles in the context of soft sets: menger spaces," Soft Computing, vol. 25, pp. 12693-12702, 2021.

[18] S. Das and S. K. Samanta, "Soft metric," Annals of Fuzzy Mathematics and Informatics, vol. 6, no. 1, pp. 77-94, 2013.

[19] S. Nazmul and S. K. Samanta, "Neighbourhood properties of soft topological spaces," Annals of Fuzzy Mathematics and Informatics, vol. 6, no. 1, pp. 1-15, 2013.

[20] S. A. El-Sheikh and A. M. Abd El-Latif, "Decompositions of some types of supra soft sets and soft continuity," International Journal of Mathematics Trends and Technology, vol. 9, pp. 37-56, 2014.

[21] T. M. Al-shami, "New soft structure: infra soft topological spaces," Mathematical Problems in Engineering, vol. 2021, Article ID 3361604, 12 pages, 2021.

[22] J. Thomas and J. S. John, "On soft generalized topological spaces," Journal of New Results in Science, vol. 4, pp. 1-15, 2014.

[23] T. M. Al-shami, "Infra soft compact spaces and application to fixed point theorem," Journal of Function Spaces, vol. 2021, Article ID 3417096, 9 pages, 2021.

[24] T. M. Al-shami and E.-S. A. Abo-Tabl, "Connectedness and local connectedness on infra soft topological spaces," Mathematics, vol. 9, no. 15, p. 1759, 2021.
[25] B. M. Ittanagi, "Soft bitopological spaces," International Journal of Computer Applications, vol. 107, no. 7, pp. 1-4, 2014.

[26] C. G. Aras and S. Bayramov, "Results of some separation axioms in supra soft topological spaces," TWMS Journal of Pure and Appllied Mathematics, vol. 9, no. 1, pp. 58-63, 2019.

[27] M. E. El-Shafei, M. Abo-Elhamayel, and T. M. Al-shami, "Partial soft separation axioms and soft compact spaces," Filomat, vol. 32, no. 13, pp. 4755-4771, 2018.

[28] S. Bayramov and C. G. Aras, "A new approach to separability and compactness in soft topological spaces," TWMS Journal of Pure and Applied Mathematics, vol. 9, no. 1, pp. 82-93, 2018.

[29] M. Terepeta, "On separating axioms and similarity of soft topological spaces," Soft Computing, vol. 23, no. 3, pp. 1049-1057, 2019.

[30] A. Singh and N. S. Noorie, "Remarks on soft axioms," Annals of Fuzzy Mathematics and Informatics, vol. 14, no. 5, pp. 503-513, 2017.

[31] F. Feng, Y. M. Li, B. Davvaz, and M. I. Ali, "Soft sets combined with fuzzy sets and rough sets: a tentative approach," Soft Computing, vol. 14, pp. 899-911, 2010.

[32] A. Kharal and B. Ahmed, "Mappings on soft classes," New Mathematic Natural Computing, vol. 7, no. 3, pp. 471-481, 2011.

[33] T. M. Al-shami, "Homeomorphism and quotient mappings in infra soft topological spaces," Journal of Mathematics, vol. 2021, Article ID 3388288, 10 pages, 2021.

[34] M. E. A. E. Monsef, O. A. Embaby, and M. K. E. Bably, "Comparison between rough set approximations based on different topologies," International Journal of Granular Computing, Rough Sets and Intelligent Systems, vol. 3, no. 4, pp. 292-305, 2014.

[35] W. S. Amer, M. I. Abbas, and M. K. El-Bably, "On j-near concepts in rough sets with some applications," Journal of Intelligent and Fuzzy Systems, vol. 32, no. 1, pp. 1089-1099, 2017.

[36] A. Azzam, A. M. Khalil, and S.-G. Li, "Medical applications via minimal topological structure," Journal of Intelligent and Fuzzy Systems, vol. 39, no. 3, pp. 4723-4730, 2020. 